\title{
Minimally invasive aortic valve replacement provides equivalent outcomes at reduced cost compared with conventional aortic valve replacement: A real-world multi-institutional analysis
}

\author{
Ravi K. Ghanta, MD, ${ }^{a}$ Damien J. Lapar, MD, MSc, ${ }^{a}$ John A. Kern, MD, ${ }^{a}$ Irving L. Kron, MD, ${ }^{a}$ \\ Alan M. Speir, MD, ${ }^{b}$ Edwin Fonner, Jr, DrPH, ${ }^{\mathrm{c}}$ Mohammed Quader, MD, ${ }^{\mathrm{d}}$ and Gorav Ailawadi, $\mathrm{MD}^{\mathrm{a}}$
}

\begin{abstract}
Background: Several single-center studies have reported excellent outcomes with minimally invasive aortic valve replacement (mini-AVR). Although criticized as requiring more operative time and complexity, mini-AVR is increasingly performed. We compared contemporary outcomes and cost of mini-AVR versus conventional AVR in a multi-institutional regional cohort. We hypothesized that mini-AVR provides equivalent outcomes to conventional AVR without increased cost.
\end{abstract}

Methods: Patient records for primary isolated AVR (2011-2013) were extracted from a regional, multi-institutional Society of Thoracic Surgeons database and stratified by conventional versus mini-AVR, performed by either partial sternotomy or right thoracotomy. To compare similar patients, a 1:1 propensitymatched cohort was performed after adjusting for surgeon; operative year; and Society of Thoracic Surgeons risk score, including age and risk factors ( $\mathrm{n}=289$ in each group). Differences in outcomes and cost were analyzed.

Results: A total of 1341 patients underwent primary isolated AVR, of which 442 $(33 \%)$ underwent mini-AVR at 17 hospitals. Mortality, stroke, renal failure, and other major complications were equivalent between groups. Mini-AVR was associated with decreased ventilator time ( 5 vs 6 hours; $P=.04$ ) and decreased blood product transfusion $(25 \%$ vs $32 \% ; P=.04)$. A greater percentage of mini-AVR patients were discharged within 4 days of the operation $(15.2 \%$ vs $4.8 \%$; $P<.001)$. Consequently, total hospital costs were lower in the mini-AVR group $(\$ 36,348$ vs $\$ 38,239 ; P=.02)$.

Conclusions: Mortality and morbidity outcomes of mini-AVR are equivalent to conventional AVR. Mini-AVR is associated with decreased ventilator time, blood product use, early discharge, and reduced total hospital cost. In contemporary clinical practice, mini-AVR is safe and cost-effective. (J Thorac Cardiovasc Surg 2015;149:1060-5)

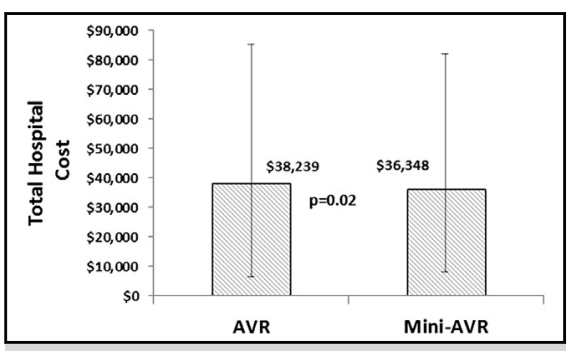

Median total hospital cost was $\$ 1891(P=.02)$ lower in mini-AVR compared with conventional AVR.

\section{Central Message}

Mortality and morbidity outcomes of mini-AVR are equivalent to conventional AVR in a real-world multi-institutional analysis. Mini-AVR is associated with decreased ventilator time, blood product use, early discharge, and reduced total hospital cost. In contemporary clinical practice, mini-AVR is safe and cost-effective.

\section{Perspective}

Previous studies evaluating mini-AVR have been primarily limited to single high-volume centers. The contemporary outcomes and cost of mini-AVR as it has disseminated to centers to real-world clinical practice is unknown. In this study, we compare contemporary outcomes and costs mini-AVR and conventional AVR outcomes and cost in a multi-institutional regional cohort. Mini-AVR is associated with decreased ventilator time, blood product use, early discharge, and reduced total hospital cost. In contemporary clinical practice, mini-AVR is safe and cost-effective.

See Editorial Commentary page 1066.

\footnotetext{
From the Division of Thoracic and Cardiovascular Surgery, ${ }^{\mathrm{a}}$ Department of Surgery, University of Virginia, Charlottesville, Va; Cardiovascular and Thoracic Associa-

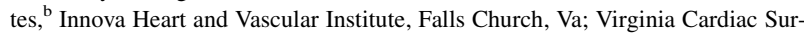
gery Quality Initiative, ${ }^{\mathrm{c}}$ Richmond, Va; and Division of Cardiothoracic Surgery, ${ }^{\mathrm{d}}$ Department of Surgery, Virginia Commonwealth University, Richmond, Va.

Supported by the Department of Surgery, University of Virginia, Charlottesville, Va.

Received for publication Nov 15, 2014; revisions received Dec 30, 2014; accepted for publication Jan 6, 2015; available ahead of print Feb 11, 2015.

Address for reprints: Gorav Ailawadi, MD, Division of Thoracic and Cardiovascular Surgery, Department of Surgery, University of Virginia School of Medicine, PO Box 800679, Charlottesville, VA 22908 (E-mail: gorav@ virginia.edu).

$0022-5223 / \$ 36.00$

Copyright (c) 2015 by The American Association for Thoracic Surgery

http://dx.doi.org/10.1016/j.jtcvs.2015.01.014
}

Aortic valve replacement (AVR) via a full sternotomy has long been the standard approach to treat aortic valve pathology and can be performed with minimal morbidity and mortality. ${ }^{1}$ Introduced in the 1990s, minimally invasive aortic valve replacement (mini-AVR), performed via a partial upper sternotomy or right thoracotomy, is increasingly performed at cardiac surgery centers across the United States. ${ }^{2}$ Several groups have demonstrated that mini-AVR can be performed with excellent outcomes, less patient discomfort, decreased blood product transfusion, and reduced length of stay (LOS) compared with conventional AVR. ${ }^{3-7}$ Mini-AVR has been criticized for increased cardiopulmonary bypass, crossclamp, and operating room 


$$
\begin{aligned}
& \text { Abbreviations and Acronyms } \\
& \begin{aligned}
\text { AVR } & =\text { aortic valve replacement } \\
\text { ICU } & =\text { intensive care unit } \\
\text { LOS } & =\text { length of stay } \\
\text { Mini-AVR }= & \text { minimally invasive aortic valve } \\
& \text { replacement } \\
\text { PROM } & \text { predicted risk of mortality } \\
\text { PROMM } & =\text { predicted risk of morbidity and } \\
& \text { mortality } \\
\text { STS } & =\text { Society of Thoracic Surgeons } \\
\text { VCSQI } & \text { Virginia Cardiac Surgery Quality } \\
& \text { Initiative }
\end{aligned}
\end{aligned}
$$

time, and higher cost. ${ }^{8,9}$ Because partial sternotomy or thoracotomy provide limited exposure to the heart, myocardial protection, de-airing, and valve exposure can be more challenging. ${ }^{10}$ Previous published studies on miniAVR have been limited to single, high-volume centers. The contemporary outcomes of mini-AVR as it has disseminated to centers with more diverse volume and experience is unknown. Furthermore, no previous study has evaluated outcomes and cost of mini-AVR in a multi-institutional cohort.

In this study, we compare contemporary mini-AVR and conventional AVR outcomes and cost in a multiinstitutional regional cohort. We hypothesize that mini-AVR provides equivalent outcomes to conventional AVR without increased cost.

\section{PATIENTS AND METHODS}

The Virginia Cardiac Surgery Quality Initiative (VCSQI) is a voluntary group of 17 hospitals and 13 cardiac surgical practices providing cardiac surgery in the Commonwealth of Virginia. VCSQI members perform more than $99 \%$ of the commonwealth's cardiac surgery procedures. The VCSQI data registry is a Society of Thoracic Surgeons (STS) certified database (version 2.73). This investigation was exempt from formal institutional review board review at each participating center because it represents a secondary analysis of the VCSQI data registry with the absence of Health Insurance Portability and Accountability Act patient identifiers and because the data are collected for quality analysis and purposes other than research.

\section{Patients and Data Acquisition}

Starting in 2011, operative approach for AVR was recorded within the VCSQI database, allowing for identification of minimally invasive procedures. De-identified patient records for all patients who underwent isolated primary AVR for the study period of January 1, 2011, to December 31, 2013, were obtained from the VCSQI registry. Exclusion criteria included endocarditis, reoperative status, and any other concomitant surgical procedure. Patient records were then stratified by operative approach: full sternotomy, partial-sternotomy, or right thoracotomy. Patients undergoing full sternotomy were placed in the conventional AVR group. Patients undergoing partial sternotomy and right thoracotomy were combined into the mini-AVR group. Patient preoperative, operative, and postoperative variables were retrieved from the VCSQI database for each patient. STS predicted risk of morbidity and mortality (PROMM) and predicted risk of mortality (PROM) were individually calculated.

\section{Cost Data and Acquisition}

The VCSQI data registry combines standardized clinical data extracted from the STS data entry forms with hospital inpatient discharge financial data. Hospital inpatient data from UB-92 and UB-04 files are matched with each STS patient record. By the use of center-specific cost-tocharge ratios, estimated hospital costs are determined with previously described methods. ${ }^{11,12}$ VCSQI maintains a $99 \%$ matching rate between STS patient records and billing data.

\section{Measured Outcomes}

The primary outcomes were frequency of postoperative complications, LOS, operative mortality, and hospital cost. Operative mortality was defined as all patient deaths occurring during hospitalization as well as those within 30 days of the date of surgery despite discharge status. Ventilation time, intensive care unit (ICU) hours, and hospital LOS from surgery to discharge were measured. Early discharge was defined as discharge by the fourth postoperative day. Standard STS definitions for postoperative events and complications were used, including cerebrovascular accident, renal failure (increase in serum creatinine level $>2.0$ or doubling of the most recent preoperative creatinine), prolonged ventilation ( $>24$ hours of mechanical ventilation), presence of any new onset atrial fibrillation, deep sternal wound infection, and administration of intraoperative or postoperative blood product. ${ }^{11}$

\section{Statistical Analysis}

All study group comparisons were unpaired. Categorical variables were compared using either Pearson $\chi^{2}$ or Fisher exact tests, and continuous variables were compared using the Student $t$ test for normally distributed data or the Wilcoxon rank sum test for nonnormally distributed data where appropriate. Propensity score matching was performed to generate a study cohort of matched patients undergoing conventional AVR and mini-AVR adjusted for potential confounding. Propensity scores were estimated using binary logistic regression models with performance of mini-AVR as the response variable and STS PROM, operative year, and operating surgeon as possible confounding predictor variables. Propensity scores were then used to match conventional AVR and mini-AVR patients in a 1:1 ratio using nearest neighbor greedy methodology, resulting in equal study cohorts. Postoperative outcomes were then compared between matched groups using standard univariate statistical tests of association.

\section{RESULTS \\ Patient Characteristics}

In our regional cohort, a total of 1341 patients underwent isolated AVR during the study period, of whom $442(33 \%)$ underwent mini-AVR at 17 hospitals. Patient characteristics for the overall cohort are reported in Table 1 and grouped by surgical approach. Mini-AVR patients were older (aged 74 years vs 69 years; $P<.001$ ) and had a greater incidence of peripheral vascular disease $(12.7 \%$ vs $8.0 \% ; P<.001)$ and end-stage renal disease $(3.6 \%$ vs $1.3 \% ; P<.01)$. In the unmatched cohort, conventional AVR patients had a higher STS PROM $(1.8 \%$ vs $1.3 \% ; P<.001)$ and STS PROMM $(14.2 \%$ vs $11.8 \% ; P<.001)$. Moderate or severe aortic insufficiency, which some groups use as a relative contraindication for mini-AVR, was present in $26.2 \%(n=116)$ of patients undergoing mini-AVR. There were no statistically significant differences in other comorbidities such as hypertension, chronic obstructive pulmonary disease, diabetes, or heart failure between the 2 unmatched groups. The patient 
TABLE 1. Characteristics for overall unmatched cohort in patients undergoing conventional aortic valve replacement (AVR) and minimally invasive aortic valve replacement (mini-AVR)

\begin{tabular}{|c|c|c|c|}
\hline Characteristic & $\begin{array}{c}\text { Conventional } \\
\text { AVR }(n=899)\end{array}$ & $\begin{array}{c}\text { Mini-AVR } \\
(n=442)\end{array}$ & $\begin{array}{c}P \\
\text { value }\end{array}$ \\
\hline Age & $69(60,77)$ & $74(63,83)$ & $<.01$ \\
\hline Female gender & $378(42.0)$ & $201(45.5)$ & .233 \\
\hline Body surface area & $2.0(1.9,2.3)$ & $2.0(1.8,2.2)$ & $<.01$ \\
\hline Hypertension & $713(79.3)$ & $348(78.7)$ & .81 \\
\hline Diabetes & $299(33.3)$ & $132(29.9)$ & .06 \\
\hline Peripheral vascular disease & $72(8.0)$ & $56(12.7)$ & $<.01$ \\
\hline End-stage renal disease & $12(1.3)$ & $16(3.6)$ & $<.01$ \\
\hline Chronic lung disease & $242(26.9)$ & $139(31.4)$ & .06 \\
\hline Mild & $137(15.2)$ & $65(14.7)$ & \\
\hline Moderate & $60(6.7)$ & $35(7.9)$ & \\
\hline Severe & $45(5.0)$ & $39(8.8)$ & \\
\hline Congestive heart failure & $368(40.9)$ & $192(43.4)$ & .29 \\
\hline NYHA I & $15(1.7)$ & $9(2.0)$ & \\
\hline NYHA II & $137(15.2)$ & $58(13.1)$ & \\
\hline NYHA III & $153(17.0)$ & $81(18.3)$ & \\
\hline NYHA III & $53(7.0)$ & $44(10.0)$ & \\
\hline $\begin{array}{l}\text { Left ventricular ejection } \\
\text { fraction }(\%)\end{array}$ & $60(53,63)$ & $60(55,64)$ & .72 \\
\hline $\begin{array}{l}\text { Moderate or severe aortic } \\
\text { insufficiency }\end{array}$ & $246(27.4)$ & $116(26.2)$ & .79 \\
\hline STS PROMM (\%) & $14.2(10.1,20.0)$ & $11.8(9.0,17.1)$ & $<.01$ \\
\hline STS PROM $(\%)$ & $1.8(1.0,3.1)$ & $1.3(0.8,2.3)$ & $<.01$ \\
\hline \multicolumn{4}{|c|}{$\begin{array}{l}\text { Boldface type indicates statistically significant findings }(P<.05) \text {. Values are } \\
\text { presented as n }(\%) \text {, or for continuous variables, as median }(25 \text { th, } 75 \text { th percentiles }) \text {. } \\
\text { NYHA, New York Heart Association functional class; STS PROMM, Society of } \\
\text { Thoracic Surgery Predicted Risk of Morbidity or Mortality; STS PROM, Society } \\
\text { of Thoracic Surgery Predicted Risk of Mortality. }\end{array}$} \\
\hline
\end{tabular}

characteristics of the propensity-score-matched groups are shown in Table 2. After 1:1 matching by surgeon, operative year, and STS PROM, patients undergoing conventional AVR and mini-AVR shared similar preoperative comorbidities. The groups had equivalent STS PROM and PROMM.

\section{Operative Characteristics}

Mini-AVR was performed via a partial sternotomy $(\mathrm{n}=410 ; 93 \%)$ or right thoracotomy $(\mathrm{n}=32 ; 7 \%)$. Median cardiopulmonary bypass time (104 vs 103 minutes; $P=.64$ ) and ischemic time (75 vs 73 minutes; $P=.61$ ) was equivalent between the 2 groups. Central arterial and venous cannulation was the preferred perfusion technique in the majority of cases $(\mathrm{n}=311 ; 70 \%)$. Femoral venous cannulation was performed in $131(30 \%)$ patients and femoral arterial cannulation was performed in 78 patients $(18 \%)$.

\section{Outcomes of Matched Cohorts}

Operative morbidity, mortality, and LOS are reported in Table 3 and stratified by surgical approach for propensitymatched patients. Operative mortality was equivalent between the 2 groups $(0.3 \%$ mini-AVR vs $2.1 \%$ AVR; $P=.06)$. There were no statistically significant differences in the rates of postoperative stroke ( $1.0 \%$ mini-AVR vs $2.1 \%$ AVR; $P=.37)$,
TABLE 2. Characteristics for propensity score-matched cohort in patients undergoing conventional aortic valve replacement (AVR) and minimally invasive aortic valve replacement (mini-AVR)

\begin{tabular}{|c|c|c|c|}
\hline Characteristic & $\begin{array}{c}\text { Conventional } \\
\text { AVR }(\mathbf{n}=\mathbf{2 8 9})\end{array}$ & $\begin{array}{c}\text { Mini-AVR } \\
(\mathbf{n}=\mathbf{2 8 9})\end{array}$ & $\begin{array}{c}P \\
\text { value }\end{array}$ \\
\hline Age & $67(58,74)$ & $67(60,76)$ & .36 \\
\hline Female gender & $110(38.1)$ & $103(35.6)$ & .55 \\
\hline Body surface area & $2.1(1.9,2.3)$ & $2.0(1.9,2.2)$ & .41 \\
\hline Hypertension & 225 (77.9) & $215(74.4)$ & .33 \\
\hline Diabetes & $93(32.2)$ & $76(26.3)$ & .12 \\
\hline Peripheral vascular disease & $22(7.6)$ & $25(8.7)$ & .65 \\
\hline End-stage renal disease & $7(2.4)$ & $3(1.0)$ & .20 \\
\hline Chronic lung disease & $67(23.2)$ & $65(22.5)$ & .57 \\
\hline Mild & $42(14.5)$ & $33(11.4)$ & \\
\hline Moderate & $15(5.2)$ & $18(6.2)$ & \\
\hline Severe & $10(3.5)$ & $14(4.8)$ & \\
\hline Congestive heart failure & $196(67.8)$ & $201(69.6)$ & .31 \\
\hline NYHA I & $5(1.7)$ & $5(1.7)$ & \\
\hline NYHA II & $35(12.1)$ & $35(12.1)$ & \\
\hline NYHA III & $37(12.8)$ & $41(14.2)$ & \\
\hline NYHA III & $16(5.5)$ & $6(2.1)$ & \\
\hline $\begin{array}{l}\text { Left ventricular ejection } \\
\text { fraction }(\%)\end{array}$ & $60(53,64)$ & $60(55,65)$ & .71 \\
\hline $\begin{array}{l}\text { Moderate or severe aortic } \\
\text { insufficiency }\end{array}$ & $87(30.1)$ & $85(29.4)$ & .23 \\
\hline STS PROMM (\%) & $12.5(9.4,16.9)$ & $11.8(9.0,17.5)$ & .34 \\
\hline STS PROM (\%) & $1.5(0.9,2.4)$ & $1.4(0.8,2.3)$ & .31 \\
\hline
\end{tabular}

renal failure (1.0\% mini-AVR vs $0.7 \%$ AVR; $P=.66)$, atrial fibrillation ( $32.2 \%$ mini-AVR vs $28.4 \%$ AVR; $P=.6$ ), reoperation for bleeding $(5.2 \%$ mini-AVR vs $2.1 \%$ AVR; $P=.12)$, or respiratory insufficiency $(9.0 \%$ mini AVR vs $8.7 \%$ AVR; $P=.69)$. Ventilator time was lower in patients undergoing mini-AVR (5 vs 6 hours; $P=.04$ ) There was no significant difference in ICU LOS (43 vs 45 hours; $P=.08$ ), or hospital LOS (5.0 vs 6.0 days; $P=.10$ ) between the 2 groups. Early discharge (defined by discharge by the fourth postoperative day) was more common in patients undergoing mini-AVR $(15.8 \%$ vs $4.2 \% ; P<.01)$.

\section{Blood Product Transfusion}

In matched patients, fewer patients undergoing miniAVR received a blood product transfusion compared with those undergoing conventional AVR $(24.6 \%$ vs $31.8 \%$; $P=.05)$. Mini-AVR patients received $47 \%$ fewer units of blood on average ( 0.28 vs 0.53 units packed red blood cells; $P=.008)$.

\section{Cost}

Hospital cost data for the matched groups is reported in Figure 1. Total hospital cost was lower in the mini-AVR group $(\$ 36,348$ vs $\$ 38,239 ; P=.02)$. 
TABLE 3. Operative outcomes in propensity-matched patients undergoing conventional aortic valve replacement (AVR) and minimally invasive aortic valve replacement (mini-AVR)

\begin{tabular}{lccr}
\hline \multicolumn{1}{c}{ Outcome } & $\begin{array}{c}\text { Conventional } \\
\text { AVR }(\mathbf{n}=\mathbf{2 8 9})\end{array}$ & $\begin{array}{c}\text { Mini-AVR } \\
(\mathbf{n}=\mathbf{2 8 9})\end{array}$ & $\begin{array}{c}\boldsymbol{P} \\
\text { value }\end{array}$ \\
\hline Death & $6(2.1)$ & $1(0.3)$ & .06 \\
Stroke & $6(2.1)$ & $3(1.0)$ & .37 \\
Renal failure & $2(0.7)$ & $3(1.0)$ & .66 \\
Atrial fibrillation & $82(28.4)$ & $93(32.2)$ & .60 \\
Reoperation for bleed & $6(2.1)$ & $15(5.2)$ & .12 \\
Blood product transfusion & $92(31.8)$ & $71(24.6)$ & $\mathbf{. 0 5}$ \\
Respiratory insufficiency & $25(8.7)$ & $26(9.0)$ & .69 \\
Ventilator time (h) & $6(4,13)$ & $5(4,11)$ & $\mathbf{. 0 4}$ \\
Intensive care unit length of stay (h) & $45(26,75)$ & $43(24,75)$ & .08 \\
Hospital length of stay (d) & $6(5,8)$ & $5(4,8)$ & .10 \\
Discharged by postoperative day 4 & $14(4.8)$ & $44(15.2)$ & $<. \mathbf{0 1}$ \\
\hline Boldface type indicates statistically significant findings $(P<.05)$. Values are presented \\
as n (\%), or for continuous variables as median $(25$ th, 75 th percentiles).
\end{tabular}

\section{DISCUSSION}

Mini-AVR surgery is increasingly desired by patients. The appeal of minimally invasive surgery is clear given its improved cosmesis, potential for less pain, and quicker recovery. ${ }^{5}$ Minimally invasive operations may be especially beneficial for specific subgroups, such as the elderly or patients with significant respiratory disease. ${ }^{13}$ Concerns that less-invasive surgery is associated greater operative complexity with longer crossclamp and bypass times are appropriate, particularly if this leads to increased morbidity and mortality. Poorer outcomes obviate any perceived benefit of less invasive operations.

In our study, we compared operative outcomes and cost of mini-AVR versus conventional AVR in a multiinstitutional regional cohort. Unlike prior studies from single high volume centers, this study evaluates the outcomes from 17 institutions-allowing a real-world and contemporary analysis. Mini-AVR was associated with equivalent cardiopulmonary bypass time and crossclamp time. In propensity-matched patients, there was no difference in mortality, stroke, renal failure, and other major morbidities between mini-AVR and conventional AVR. There was a notable trend in reduced operative mortality with miniAVR in propensity-matched patients $(0.3 \%$ vs $2.1 \%$; $P=.06)$. Mini-AVR was found to have reduced ventilator time and more frequent early discharge. Moreover, miniAVR patients were also significantly less likely to receive a transfusion. Although criticized as being more costly, mini-AVR cost was less than conventional AVR, primarily due to more frequent early discharge and less blood product use.

Previous studies have also uniformly found longer crossclamp and bypass times with mini-AVR. ${ }^{3,14,15}$ The clinical significance of this is unclear, because these same studies identified no associated increase in morbidity or mortality.

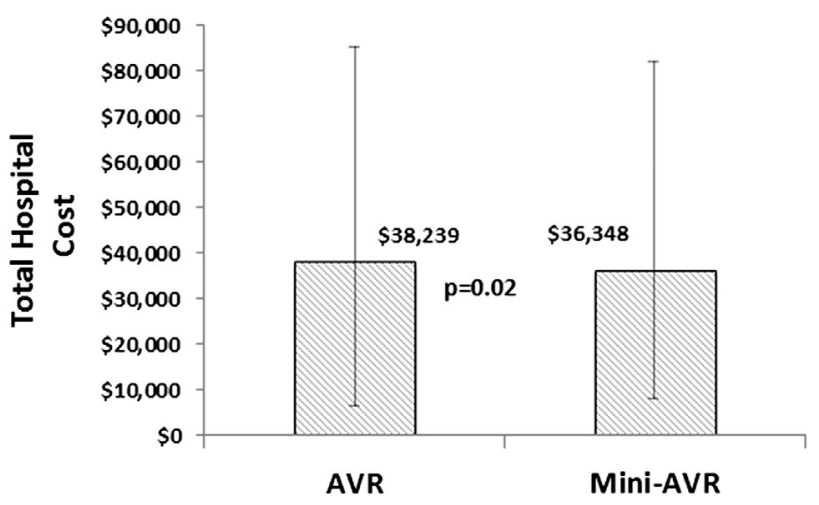

FIGURE 1. Hospital costs for propensity-matched patients. Median total hospital cost was $\$ 1891(P=.02)$ lower in minimally invasive aortic valve replacement compared with conventional aortic valve replacement. $A V R$, Aortic valve replacement; Mini-AVR, minimally invasive aortic valve replacement.

In our study, we found that crossclamp and bypass times were equal in full sternotomy and mini-AVR, suggesting that surgeons have now adopted techniques to reduce bypass and crossclamp times with mini-AVR. Mini-AVR crossclamp times are now similar to national STS data for AVR. There are, however, scenarios where minimally invasive surgery will inevitably increase operative times compared with conventional surgery. Given this, new surgeons and centers embarking on minimally invasive surgery should devise a strategy to optimize myocardial protection. New technology, such as endovascular pulmonary artery vents and cardioplegia catheters, have been developed to improve myocardial protection during mini-AVR should be considered in a minimally invasive surgeon's armamentarium. ${ }^{15}$

Consistent with national STS trends, overall operative mortality with AVR in our regional cohort was low with an observed mortality of $1.2 \% .^{1}$ This corresponded to an observed to expected mortality ratio of 0.84 . Prior studies comparing mini-AVR to full sternotomy AVR have also found equivalent operative mortality rates. Mihaljevik and colleagues $^{3}$ found an operative mortality of $2 \%$ in miniAVR versus $3 \%$ in conventional AVR in 526 patients who underwent AVR at the Brigham and Women's Hospital. Johnston and colleagues ${ }^{5}$ found an identical $0.96 \%$ operative mortality in 832 propensity-matched patients at the Cleveland Clinic. Brown and colleagues ${ }^{14}$ performed a meta-analysis of 26 studies and 4586 patients undergoing mini-AVR versus conventional AVR. They found no difference in operative mortality (odds ratio, $0.71 ; 95 \%$ confidence interval, 0.49-1.02).

Corresponding with prior studies, there was no significant difference in major morbidity (eg, stroke and renal failure) between mini-AVR and conventional AVR. Similar to previous studies, mini-AVR decreased median ventilator time by $17 \%$ ( 1 hour). Johnston and colleagues ${ }^{5}$ reported a median ventilator time of 5.2 hours for mini-AVR versus 
6.9 hours for conventional AVR. Many high-volume minimally invasive valve centers perform early extubation in the operating room. Such strategies may reduce ventilator times and LOS and further widen the difference between mini- and conventional AVR. ${ }^{16}$ Mirroring national STS trends, postoperative LOS is decreasing after AVR. In 2006, median postoperative LOS was 7.06 days. By comparison, in our study postoperative LOS was 5 days (mini-AVR) and 6 days (conventional AVR). Although median ICU and hospital LOS were statistically equivalent, patients undergoing mini-AVR were 3 times more frequently discharged by the fourth postoperative day. Earlier discharge provides a potential opportunity for improved resource use and patient satisfaction.

As noted in prior studies, mini-AVR is associated with decreased blood product use. ${ }^{3,5,7,17-19}$ Twenty five percent of mini-AVR patients received blood products compared with $32 \%$ for conventional AVR, with mini-AVR patients receiving $47 \%$ fewer units of packed red blood cells on average. Numerous studies have indicated that transfusion is associated with worse short- and long-term outcomes following cardiac surgery. ${ }^{20,21}$ Reduced transfusion reduces resource use and may provide benefits beyond inhospital outcomes. ${ }^{11}$

Few studies have evaluated the cost of mini-AVR, which has been criticized as more expensive due to longer operative times and need for specialized equipment. In our study, we found that total hospital costs were $5 \%$ lower in patients who underwent mini-AVR compared with conventional AVR. The primary reason for lower total costs were more frequent early discharge and lower blood product use. Prior single-center studies have reported decreased cost with mini-AVR. Cohn and colleagues ${ }^{22}$ reported that miniAVR reduced hospital charges by $\$ 7000$. Cosgrove and colleagues $^{23}$ reported that mini-AVR reduced hospital direct costs by $19 \%$.

Most prior studies have been limited to a single center's experience with mini-AVR. In our study, mini-AVR was performed at 17 hospitals. We believe the results of this study are more reflective of contemporary clinical practice. Mini-AVR represented 33\% of total isolated AVR volume in the Commonwealth of Virginia. The median operative volume was 4.5 cases per hospital over the 3-year study period. The results of this study indicate that equivalent outcomes to conventional AVR can be obtained at centers with diverse experience and volume.

This study does have some important limitations. It is a retrospective analysis. We do not know the selection criteria that individual surgeons used to select patients for miniAVR. We used propensity score matching to create similar AVR and mini-AVR cohorts for comparison. We intentionally limited our propensity matching variables to the STS PROM score, surgeon, and operative year to avoid overestimation with modeling. Although using this technique 35\% of mini-AVR patients were unmatched and thus not included for comparison, we were able to identify sizeable, well-matched cohorts. We included both partial sternotomy and right thoracotomy approaches for mini-AVR because we wanted to include all real-world minimally invasive approaches in this analysis. Because the right thoracotomy approach was limited to a small number of patients, the outcomes of this study are unaffected by their inclusion. Comparison of partial sternotomy and right thoracotomy would be an important future study; however, we did not have adequate sample size for this analysis in our cohort. Important outcome data such as postoperative pain, patient satisfaction, and return to work were also not captured in our database. These are the primary rationale to perform mini-AVR and others have demonstrated superiority to conventional AVR. ${ }^{22,24}$ Cost data were limited to in-hospital costs. Additional factors, such as rehabilitation cost and lost productivity, were not included in the cost analysis. Including these health-care costs may have increased overall savings with mini-AVR compared with conventional AVR.

\section{CONCLUSIONS}

The outcomes of mini-AVR are equivalent to conventional AVR in a contemporary multi-institutional analysis. Mini-AVR is associated with decreased ventilator time, blood product use, early discharge, and reduced total hospital cost. In contemporary clinical practice, miniAVR is safe and cost-effective.

\section{Conflict of Interest Statement}

Alan M. Speir reports consulting fees from Medtronic. All other authors have nothing to disclose with regard to commercial support.

\section{References}

1. Brown JM, O'Brien SM, Wu C, Sikora JA, Griffith BP, Gammie JS. Isolated aortic valve replacement in North America comprising 108,687 patients in 10 years: changes in risks, valve types, and outcomes in the Society of Thoracic Surgeons National Database. J Thoracic Cardiovasc Surg. 2009;137:82-90.

2. Cosgrove DM III, Sabik JF. Minimally invasive approach for aortic valve operations. Ann Thorac Surg. 1996;62:596-7.

3. Mihaljevic T, Cohn LH, Unic D, Aranki SF, Couper GS, Byrne JG. One thousand minimally invasive valve operations: early and late results. Ann Surg. 2004;240: 529-34; discussion 34.

4. Tabata M, Umakanthan R, Cohn LH, Bolman RM III, Shekar PS, Chen FY, et al. Early and late outcomes of 1000 minimally invasive aortic valve operations. Eur J Cardiothorac Surg. 2008;33:537-41.

5. Johnston DR, Atik FA, Rajeswaran J, Blackstone EH, Nowicki ER, Sabik JF III, et al. Outcomes of less invasive J-incision approach to aortic valve surgery. J Thorac Cardiovasc Surg. 2012;144:852-8.e3.

6. Liu J, Sidiropoulos A, Konertz W. Minimally invasive aortic valve replacement (AVR) compared to standard AVR. Eur J Cardiothorac Surg. 1999;16(Suppl 2): S80-3.

7. Bonacchi M, Prifti E, Giunti G, Frati G, Sani G. Does ministernotomy improve postoperative outcome in aortic valve operation? A prospective randomized study. Ann Thorac Surg. 2002;73:460-5; discussion 5-6.

8. Cooley DA. Antagonist's view of minimally invasive heart valve surgery. J Cardiac Surg. 2000;15:3-5. 
9. Cunningham MJ, Berberian CE, Starnes VA. Is transthoracic minimally invasive aortic valve replacement too time-consuming for the busy cardiac surgeon? Innovations. 2011;6:10-4.

10. Tabata M, Umakanthan R, Khalpey Z, Aranki SF, Couper GS, Cohn LH, et al. Conversion to full sternotomy during minimal-access cardiac surgery: reasons and results during a 9.5-year experience. J Thorac Cardiovasc Surg. 2007;134: $165-9$.

11. LaPar DJ, Crosby IK, Ailawadi G, Ad N, Choi E, Spiess BD, et al. Blood product conservation is associated with improved outcomes and reduced costs after cardiac surgery. J Thorac Cardiovasc Surg. 2013;145:796-803; discussion 804.

12. Speir AM, Kasirajan V, Barnett SD, Fonner E Jr. Additive costs of postoperative complications for isolated coronary artery bypass grafting patients in Virginia. Ann Thorac Surg. 2009;88:40-5; discussion 805-6.

13. ElBardissi AW, Shekar P, Couper GS, Cohn LH. Minimally invasive aortic valve replacement in octogenarian, high-risk, transcatheter aortic valve implantation candidates. J Thorac Cardiovasc Surg. 2011;141:328-35.

14. Brown ML, McKellar SH, Sundt TM, Schaff HV. Ministernotomy versus conventional sternotomy for aortic valve replacement: a systematic review and metaanalysis. J Thorac Cardiovasc Surg. 2009;137:670-9.e5.

15. Brinkman WT, Hoffman W, Dewey TM, Culica D, Prince SL, Herbert MA, et al. Aortic valve replacement surgery: comparison of outcomes in matched sternotomy and PORT ACCESS groups. Ann Thorac Surg. 2010;90:131-5.

16. Zhu F, Lee A, Chee YE. Fast-track cardiac care for adult cardiac surgical patients. Cochrane Database Syst Rev. 2012;10:CD003587.
17. Bakir I, Casselman FP, Wellens F, Jeanmart H, De Geest R, Degrieck I, et al Minimally invasive versus standard approach aortic valve replacement: a study in 506 patients. Ann Thorac Surg. 2006;81:1599-604.

18. Dogan S, Dzemali O, Wimmer-Greinecker G, Derra P, Doss M, Khan MF, et al. Minimally invasive versus conventional aortic valve replacement: a prospective randomized trial. J Heart Valve Dis. 2003;12:76-80.

19. Machler HE, Bergmann P, Anelli-Monti M, Dacar D, Rehak P, Knez I, et al. Minimally invasive versus conventional aortic valve operations: a prospective study in 120 patients. Ann Thorac Surg. 1999;67:1001-5.

20. Murphy GJ, Reeves BC, Rogers CA, Rizvi SI, Culliford L, Angelini GD Increased mortality, postoperative morbidity, and cost after red blood cell trans fusion in patients having cardiac surgery. Circulation. 2007;116:2544-52.

21. Hajjar LA, Vincent JL, Galas FR, Nakamura RE, Silva CM, Santos MH, et al Transfusion requirements after cardiac surgery: the TRACS randomized controlled trial. JAMA. 2010;304:1559-67.

22. Cohn LH, Adams DH, Couper GS, Bichell DP, Rosborough DM, Sears SP, et al Minimally invasive cardiac valve surgery improves patient satisfaction while reducing costs of cardiac valve replacement and repair. Ann Surgery. 1997; 226:421-6; discussion 7-8.

23. Cosgrove DM III, Sabik JF, Navia JL. Minimally invasive valve operations. Ann Thorac Surg. 1998;65:1535-8; discussion 8-9.

24. Candaele S, Herijgers P, Demeyere R, Flameng W, Evers G. Chest pain after partial upper versus complete sternotomy for aortic valve surgery. Acta Cardiologica. 2003;58:17-21.

Readers who found these articles interesting may also like to read the following papers found in recent and future issues of our sister publications, Seminars in Thoracic and Cardiovascular Surgery and Operative Techniques in Thoracic and Cardiovascular Surgery!

Current Readings: Tirone David. Aortic Valve Sparing Operations. Semin Thorac Cardiovasc Surg. Autumn 2014;26(3):231-238.

News and Views: Rakesh Suri. Mitral Valve Repair in Asymptomatic Patients with Severe Mitral Regurgitation: Pushing Past the Tipping Point. Semin Thorac Cardiovasc Surg. Summer 2014;26(2):95-101.

News and Views: Michael Reardon. The CoreValve US Pivotal Trial. Semin Thorac Cardiovasc Surg. Autumn 2014;26(3):179-86.

Ravi Ghanta. Staged Hybrid Repair for Extent II Thoracoabdominal Aortic Aneurysms and Dissections. Oper Tech Thorac Cardiovasc Surg. Summer 2014;19(2):238-251.

Gorav Ailawadi. Transcatheter MV repair. Oper Tech Thorac Cardiovasc Surg. Summer 2014;19(2):219-237. 